\title{
Defining computational thinking framework for introductory programming in higher education
}

\author{
Boštjan Bubnič \\ University of Maribor \\ Faculty of Electrical Engineering and Computer Science \\ bostjan.bubnic@student.um.si
}

\begin{abstract}
Computational thinking (CT) is gaining recognition as an important skill set for the general problem solving. Although CT has its origin in computer science and programming, there is a great interest of researchers and educators to explore how to include CT in all scientific and engineering disciplines, as well as in kindergarten to $12^{\text {th }}$ grade education (K-12) curriculum. To determine the effective methods for teaching, learning and assessing CT, a definition and its scope is needed. To date there is no consensus in terms of formal CT definition as well as the definitive or necessary components of CT. However, our study builds upon the consensus that multiple skills are involved in $\mathrm{CT}$. The result of this study is the CT framework proposal to be used in introductory programming courses in higher education. The framework is an intersection of previous research that has identified basic, domain independent components of CT and domain specific programming practices. We hope that the framework will encourage the future research on teaching and assessing $\mathrm{CT}$ in the higher education.
\end{abstract}

\section{Keywords}

Computetional thinking, Framework, Introductory programming, Higher education

\section{INTRODUCTION}

Computational thinking (CT) has a long history within computer science (CS). It was known under the terms "algorithmizing" and "algorithmic thinking" in the 1950s and 1960s, when it was considered as a mental practice for problem conceptualization, for inventing formalisms and concepts to solve problems [22]. In the educational context, the CT phrase was introduced by Seymour Papert in the 1980s, when he was teaching Logo, an educational programming language, to improve students's ability to think procedurally [12]. Recently, the term was reintroduced and popularized by Wing who described CT as a way of solving problems, designing systems, and understanding human behavior by drawing on the concepts fundamental to CS [25]. She also argued that computational thinking is a fundamental skill for everyone, not just for the computer scientists. The shift from the CS to the general problem solving domain increased attention among researchers, educators and practitioners in exploring how to include CT across the educational spectrum and in everyday life. However, the broad spectrum of perspectives on CT also presents challenges to teaching, learning and assessing CT. The diverse spectrum of concepts, skills and practices under the umbrella of CT resulted in the lack of clarity as to what computational thinking should be. In this regard, to date there is no consensus concerning the definition and the scope of CT, nor there is a consensus about the definitive or necessary components that constitute CT. Nevertheless, it is generally agreed that multiple skills are involved in CT [18]. Moreover, researchers have begun to characterize computational thinking by means of CT taxonomies and frameworks, where particular concepts and practices were observed.

This work expanded upon our previous work where general, domain independent components of CT were identified [4]. Moreover, the aforementioned study motivated this work. The main contribution of this paper is the definition of computational thinking framework that was sourced from the general, domain independent CT components. It is intended to be used in the higher education institutions. We envision this work to serve as a foundation for designing $\mathrm{CT}$ instruments and course materials to assess and foster computational thinking for CS majors and non-CS majors. The proposed framework is also aligned with the particular CS curriculum categories [17].

\section{RELATED WORK}

When computational thinking was reintroduced by Wing [25], abstraction and decomposition were the fundamental components. The initial set of components were later refined with automation. After initial component conceptualization, researchers have begun to characterize computational thinking by means of CT taxonomies and frameworks.

This section presents a brief description of related work with the focus on the frameworks and taxonomies that are applicable to higher education with the emphasis on CS domain. Gouws et al. [7] defined a framework of six distinct skills and practices that served as the foundation for the assessment design, where the intervention was applied to introductory computer science course (CS1) students. Billion- 
niere [1] assessed CS1 students' comprehension within abstraction, arrays of objects, and inheritance. The results of the assessment were categorized into five computational thinking concepts. Romero et al. [16] proposed a framework for evaluating CT in creative programming activities. The intervention was applied to undergraduate students of a bachelor's degree in elementary school education. Peteranetz et al. [14] report on fostering computational thinking through computational creativity based on an online course that was tailored for non-CS majors. Rambally [15] proposed a framework of CT skills and practices that were integrated into discrete structures course, which was a required course for all Information Technology majors. Finally, the two most cited papers in context of CT frameworks appear to be Brennan and Resnick [3] and Weintrop et al. [24]. Although none of the papers was aimed for the CT within higher education, there are studies that build on these particular papers in the context of higher education.

\section{PROPOSED FRAMEWORK}

Algorithm, abstraction and decomposition, previously identified as the uppermost general, domain independent CT components, served as a starting point for this study. Afterwards, each of the CT concepts were further examined within the computer programming domain. This was a twostep process. Fundamental programming concepts and practices were grouped into four categories within the first step. The initial corpus of computer programming concepts was sourced from the results of research conducted by LuxtonReilly et al. [10]. Within the literature review they identified more than sixty different computer programming concepts that were grouped into twelve master categories. To extract only concepts relevant to algorithm, abstraction and decomposition, each particular concept from [10] was further analysed with the relevant sections of the Computer Science Curricula 2013 [17] and chapters concerning the software engineering [2]. The aims of the second step were the intersection points between particular fundamental computer programming concepts and the CT concepts. Computer Science Curricula 2013 [17] and the dissertation reporting on the process of developing validated CS assessment [23] were the primary sources for the aligning process.

The proposed framework of computational thinking for introductory programming in higher education $(\mathrm{CTF})$ is presented in the Table 1. The CTF is envisioned to serve as the foundation for designing CT instruments and course materials to assess and foster computational thinking for CS majors and non-CS majors. The visual representation of the $\mathrm{CTF}$ is represented as a two dimensional grid. The axis of this framework describes the main components that make up $\mathrm{CT}$, as well as programming practices that are part of computer programming. Each grid element incorporates computer programming concepts that are aligned with particular CS curriculum category [17]. We envision each of these programming concepts to be implemented as programming artefacts within the specific programming methodology or as a pseudocode.

Algorithm, abstraction and decomposition are the relevant, domain independent CT components that were identified in our previous study [4]. It should be noted that thirty-six different CT concepts, skills and practices were identified within our previous study, while only algorithm, abstraction and decomposition appeared to be relevant, domain independent CT components. The relevance was evaluated according to the frequency identified in a literature review. Within our CTF, they are represented on the vertical axis. Algorithms can be generally defined as procedural building blocks of a computer programming, of a human thought and of a general problem solving [4]. In the theoretical computer science, an algorithm is defined as a procedure that satisfies the criteria of finiteness, input, output, effectiveness, and definiteness [8]. However, in our framework an algorithm is associated with constructing an algorithmic solution to a problem to be solved. In educational context, this practice is referred as algorithmic thinking.

Abstraction can generally be characterized as the conceptual process of eliminating specificity by ignoring certain features. Abstraction is closely related to the modeling concept and to the concept of generalization. The model is abstraction of a real or a conceptual complex system. Abstraction levels and the hierarchy of abstraction are important aspects in the models design practice. In software engineering, abstraction involves the extraction of properties of an object according to some focus: only those properties are selected which are relevant with respect to the focus [5]. In computer programming abstraction practices can be observed within two categories: by mechanisms or by programming constructs. Abstraction by specification and abstraction by parametrization are the two mechanisms that make abstractions explicit and tangible. Furthermore, these two powerful methods for constructing computer programs contribute to the definition of the following important programming constructs: procedural abstraction, data abstraction, iteration abstraction and type hierarchy.

Decomposition deals with breaking down a problem into smaller, more manageable components where each component can be managed independently. Levels of abstraction need to be utilized to successfully decompose a problem into smaller components. In computer science, distinct variants of decomposition can be observed. Parnas [13] investigated hierarchical decomposition in the context of modularity in order to decompose complex information system into a number of smaller, manageable modules. Moreover, decomposition is the crucial part of structured, object-oriented and functional programming paradigms. Generally, the aim is to decompose a computer program into modules, which are set of smaller programs to solve sub problems. Smaller programs interact with one another in a simple, well defined way.

As a discipline, computer programming incorporates several processes, skills and practices. However, our aim at this point was to identify computer programming skills and practices that correlate with particular CT concepts. The primary source for identifying programming skills and practices was the Computer Science Curricula's sections Fundamental Programming Concepts, Development Methods, Algorithms and Design [17]. Furthermore, to observe the broadest spectrum, software engineering skills and practices were also included within our study [2], [9]. Finally, problem conceptualization, implementation, debugging and evaluation were included in the framework. They are represented on horizontal axis in the Table 1. We define problem conceptualization as an algorithmic solution to the observed problem. 
Table 1: Computational thinking framework for introductory programming in higher education

\begin{tabular}{|c|c|c|c|c|}
\hline $\begin{array}{l}\text { Components of } \\
\mathrm{CT}\end{array}$ & $\begin{array}{l}\text { Problem concep- } \\
\text { tualization }\end{array}$ & Implementation & Debugging & Evaluation \\
\hline Algorithm & $\begin{array}{l}\text { - Algorithmic } \\
\text { solution to a } \\
\text { problem }\end{array}$ & $\begin{array}{l}\text { - Diagram or } \\
\text { flowchart } \\
\text { - Algorithmic } \\
\text { notions of flow } \\
\text { control }\end{array}$ & $\begin{array}{l}\text { - Debugging of } \\
\text { algorithmic } \\
\text { solution } \\
\text { - Error detec- } \\
\text { tion strategies } \\
\text { - Code compre- } \\
\text { hension }\end{array}$ & $\begin{array}{l}\text { - Correctness } \\
\text { - Efficiency } \\
\text { - Simplicity }\end{array}$ \\
\hline Abstraction & $\begin{array}{l}\text { - Abstraction levels } \\
\text { - Abstraction } \\
\text { hierarchy }\end{array}$ & $\begin{array}{l}\text { - Mechanisms of } \\
\text { abstraction } \\
\text { - Programming } \\
\text { constructs }\end{array}$ & $\begin{array}{l}\text { - Error detec- } \\
\text { tion strategies } \\
\text { - Code compre- } \\
\text { hension }\end{array}$ & $\begin{array}{l}\text { - Relevance } \\
\text { - Efficiency } \\
\text { - Simplicity }\end{array}$ \\
\hline Decomposition & $\begin{array}{l}\text { - Components } \\
\text { identification } \\
\text { - Components } \\
\text { modeling }\end{array}$ & - Modularization & $\begin{array}{l}\text { - Error detec- } \\
\text { tion strategies } \\
\text { - Code compre- } \\
\text { hension }\end{array}$ & $\begin{array}{l}\text { - Correctness } \\
\text { - Simplicity }\end{array}$ \\
\hline
\end{tabular}

It is a part of requirements engineering process that assures the problems are properly defined, understood and framed in a way that allows algorithmic solution. Domain specific knowledge is required within the requirement engineering process [2]. Implementation is a practice of programming the algorithmic solution within specific programming language. The programming language constructs principally relay on the programming methodology that is applied, such as structured programming, modular programming, abstract data type programming or object-oriented programming. Moreover, the algorithmic solution can be implemented as a pseudocode. Debugging is the process of understanding, finding and correcting errors. While various error detection strategies exist, systematic debugging strategy has proven to be the most effective [26]. To effectively locate and fix an error within a computer program, code comprehension skills are required. Donaldson and Cutts [6] proposed several activities to develop the code comprehensions skills. The evaluation process normally involves some identification of relevant standards of worth, merit or value. Moreover, the process is also concerned with some investigation of the performance of the evaluands on these standards [19]. Smith and Cordova [20] propose several traits to be used for computer program evaluation, such as correctness, efficiency and completeness.

\section{DISCUSSION}

The motivation for this work arose whilst working on previous work, where we were identifying general, domain independent components of CT [4]. During the literature review, the paper from Tang et al. [21] reporting on content analysis of computational thinking research has been examined.
Results within the paper has revealed that higher education level was the second most frequently investigated category within CT. Furthermore, computer science was the most frequent subject of research in context of CT [21]. On contrary, within our previous study we have found only a few studies investigating on the CT frameworks or taxonomies in higher education [4]. In this context, our study tried to fill the gap between $\mathrm{CT}$ concepts and computer programming concepts and practices by proposing the CTF.

The relevancy of the proposed CTF in context of CT is fundamentally different from previous studies, because only relevant, domain independent components of CT were used as a starting point of our study. It should be noted that thirtysix different CT concepts, skills and practices were identified within our previous study, while only algorithm, abstraction and decomposition appeared to be the prospects for achieving the relevancy consensus [4]. The primary aim of this study was the alignment process between these CT concepts and computer programming concepts. The mapping of the programming concepts to programming code artifacts is yet to be the subject of further research. On the contrary, previous studies that had proposed CT frameworks and taxonomies [3], [24] had mainly focused on computer programming artifacts and mapped them directly to various CT skills and practices.

Nevertheless, the major result of this work are the CTF grid elements that incorporate computer programming concepts, aligned with CS curriculum and their associated CT concepts. In this regard, the CTF is envisioned to serve as the foundation for teaching and evaluating core CT skills and practices within computer programming. 


\section{CONCLUSIONS}

The result of this work is a framework proposal for $\mathrm{CT}$ in introductory programming in higher education. The framework provides a useful starting point for further research. The future work should be oriented toward implementing computer programming artefact based on proposed CTF. The envisioned outcome of further research might be the CT instrument suited for implicitly assessing CT based on computer programming tasks within the higher education level.

Furthermore, the proposed CTF should stimulate further research in the context of success or failure of novices in introductory programming in higher education, often referred as "CS1 dropout rate". The researchers observed that the dropout rate problem can be divided to the following two categories: language problem and design problem [11]. If these categories could be mapped into the computational thinking context, then the proposed CTF could serve as a foundation for CT assessment, a potential predictor for the CS1 dropout rate.

\section{REFERENCES}

[1] E. Billionniere. Assessing Cognitive Learning of Analytical Problem Solving. Doctoral dissertation, Arizona State University, December 2011.

[2] D. Bjørner. Software Engineering 3 - Domains, Requirements, and Software Design. Springer-Verlag, Heidelberg, 2006.

[3] K. Brennan and M. Resnick. New frameworks for studying and assessing the development of computational thinking. pages 1-25, Vancouver, BC, Canada, 2012.

[4] B. Bubnic and T. Kosar. Towards a consensus about computational thinking skills: Identifying agreed relevant dimensions. Newcastle, UK, 2019. Proceedings of the 30th Annual Workshop of the Psychology of Programming Interest Group - PPIG 2019 - submited for publication.

[5] K. Czarnecki. Generative Programming: Methods, Tools, and Applications. Addison-Wesley Professional; 1 edition, June 2000.

[6] P. Donaldson and Q. Cutts. Flexible low-cost activities to develop novice code comprehension skills in schools. pages 1-4, Potsdam, Germany, 2018. ACM New York.

[7] L. Gouws, K. Bradshaw, and P. Wentworth. First year student performance in a test for computational thinking. pages 271-277, East London, South Africa, 2013. ACM New York.

[8] D. Knuth. The Art of Computer Programming: Volume 1: Fundamental Algorithms, Third Edition. Addison-Wesley, USA, 1997.

[9] A. J. Ko, R. Abraham, L. Beckwith, A. Blackwell, and M. Burnett. The state of the art in end-user software engineering. volume 43, USA, April 2011. ACM New York.

[10] A. Luxton-Reilly, B. A. Becker, Y. Cao, and R. McDermott. Developing assessments to determine mastery of programming fundamentals. pages $47-69$, July 2017.

[11] M. McCracken, V. Almstrum, D. Diaz, and M. Guzdial. A multi-national, multi-institutional study of assessment of programming skills of first-year cs students. volume 33, pages 125-180, USA, 2001. ACM New York.

[12] S. Papert. Mindstorms: children, computers, and powerful ideas. Basic Books, Inc, USA, 1980.

[13] D. Parnas. On the criteria to be used in decomposing systems into modules. volume 15, pages 1053-1058, USA, 1972. ACM.

[14] M. S. Peteranetz, L.-K. Soh, and E. Ingraham. Building computational creativity in an online course for non-majors. pages 442-448, Minneapolis, USA, 2019. ACM New York.

[15] G. Rambally. Integrating computational thinking in discrete structures. pages 99-119, Switzerland, 2017. Springer International Publishing AG.

[16] M. Romero, A. Lepage, and B. Lille. Computational thinking development through creative programming in higher education. volume 14, pages 1-15, 2017.

[17] M. Sahami, A. Danyluk, S. Fincher, and K. Fisher. Computer Science Curricula 2013. The Joint Task Force on Computing Curricula Association for Computing Machinery (ACM) IEEE Computer Society, USA, December 2013.

[18] C. Selby and J. Woollard. Refining an understanding of computational thinking. University of Southampton Institutional Repository, 2014.

[19] I. Shaw, J. Greene, and M. Mark. The SAGE Handbook of Evaluation. SAGE Publications Ltd, USA, 2013.

[20] L. Smith and J. Cordova. Weighted primary trait analysis for computer program evaluation. pages 14-19. Consortium for Computing Sciences in Colleges, 2005.

[21] K. Y. Tang, T. L. Chou, and C. C. Tsai. A content analysis of computational thinking research: An international publication trends and research typology. pages 1-11, USA, 2019. Springer Nature.

[22] M. Tedre. The long quest for computational thinking. pages 120-129. Koli, Finland, Koli Calling '16 Proceedings of the 16th Koli Calling International Conference on Computing Education Research, November 2016.

[23] A. E. Tew. Assessing Fundamental Introductory Computing Concept Knowledge in a Language Independent Manner. Doctoral dissertation, Georgia Institute of Technology, December 2010.

[24] D. Weintrop, E. Beheshti, and M. Horn. Defining computational thinking for mathematics and science classrooms. volume 25, pages 127-147, February 2016.

[25] J. Wing. Computational thinking. volume 49, pages 33-35, USA, March 2006. ACM New York.

[26] A. Zeller. Why Programs Fail: A Guide to Systematic Debugging 2nd Edition. Morgan Kaufmann, USA, June 2009. 